\title{
¿Por qué el Perú debe mantener la teleeducación en tiempos de COVID-19? Oportunidades y barreras para la educación. Retos que se avecinan
}

\author{
¿Why should Perú stand for tele-education in times of covid-19? \\ Opportunities and barriers for education. Challenges ahead
}

iD Yamil Ramos García $\unrhd$

Lima, Perú

\begin{tabular}{llll}
\hline Recibido: $11 / 07 / 2020$ & Revisado: 20/07/2020 & Aceptado: 25/09/2020 & Publicado: 25/10/2020
\end{tabular}

\section{RESUMEN}

La teleeducación es la modalidad de enseñanza-aprendizaje a distancia, flexible y abierta basada en las TICs donde los participantes cuentan con elasticidad en cómo, cuándo y dónde realizar la acción formativa. Tiene la misma validez que su equivalente tradicional y alcanza el mismo objetivo de enseñanza, logro de aprendizaje y competencias profesionales por lo que se considera la continuación de la educación y un reemplazo. Frente al COVID-19 Perú estableció un estado de emergencia sanitaria y una cuarentena estrictas con fuertes regulaciones. En el sector Educación se suspendieron las clases, el año escolar y se cerraron las instalaciones educativas. Sin embargo, la sociedad mostró la fractura del civismo. Incumplimiento de disposiciones del gobierno, inconducta en la convivencia colectiva, ignorancia sanitaria, desigualdad social, informalidad, aglomeraciones en mercados, bancos y transporte público, viviendas con familias numerosas y costumbres provocaron la multiplicación del contagio. En este ámbito la teleeducación también implica el compromiso de los actores del proceso educativo, alumnos y profesores con ambientes apropiados y acceso a medios, instalaciones educativas con recursos digitales, capacidades para analizar recursos, así como pensamiento basado en textos, imágenes, audios y videos. Por lo tanto, la teleeducación se muestra como una forma de enseñanza eficaz y de calidad para educar y proteger sanitariamente a la ciudadanía y se muestra como un medio válido y confiable para la educación en tiempos de COVID-19 manteniendo así los servicios educativos y continuar el desarrollo de individuos que aporten al bienestar y la prosperidad y el desarrollo integral de la persona en la sociedad.

Palabras clave: Perú, cuarentena, pandemia, a distancia, enseñanza, COVID-19.

\section{ABSTRACT}

Tele-education is a flexible remote teaching-learning modality, based on ICTs where the participants have elasticity in how, when and where to carry out the training action. It takes 
the same goals as its traditional equivalent and achieves the same teaching objective, learning achievement and professional competences so is considered the continuation of education. On the edge of COVID-19, Peru established a strict state of sanitary emergency and quarantine with strong regulations. In Education sector, lessons were suspended and the school year and educational facilities were closed. However, society showed the fracture of civility. Failure to comply with government regulations, misconduct in collective coexistence, health ignorance, social inequality, informality, crowds in markets, banks and public transport and homes with large families cause the multiplication of contagion. In this area, tele-education also implies the commitment of the educational process actors, students and teachers with appropriate environments and access to media, educational facilities with digital resources, capacities to analyze resources as well as thinking based on texts, images, audios and videos. Therefore, tele-education is shown as an effective and quality form of education to teach, protect and keep health, and it is shown as a valid and reliable way for education in times of COVID-19, thus maintaining educational services and continuing the development of individuals that contribute to the well-being and prosperity and the integral development of the person in society.

Keywords: Peru, quarantine, pandemic, remote, teaching, COVID-19.

\section{INTRODUCCIÓN}

La teleeducación es aquella modalidad de enseñanza-aprendizaje que está basada en las tecnologías de la información y las comunicaciones y en la que los participantes cuentan con elasticidad para decidir cómo, cuándo y dónde realizar las actividades de la acción formativa.

A diferencia de otras formas de enseñanza como las tradicionales y comúnmente presenciales, las sesiones de aprendizaje diseñadas para la teleeducación se caracterizan por ser $<<a$ distancia $>>$, con gran $<<$ flexibilidad de horario $>>$ y sobre todo $<<$ abiertas $>$.

La característica $<<a$ distancia $>>$ hace referencia a que la actividad educativa no se desarrolla dentro de un aula, laboratorio, taller, salón de clases o espacio educativo formal establecido, sino que los actores principales de la enseñanza-aprendizaje, el profesor y el estudiante, pueden encontrarse en lugares físicos diferentes y distantes y no compartir los mismos ambientes; a este rasgo se le conoce también como "no presencial".

El atributo <<flexibilidad de horario >> apunta a que la interacción entre los participantes durante la sesión de clases no requiere suceder en un mismo momento del tiempo ni tener la misma duración, de esta forma los involucrados en la actividad educativa no necesitan coincidir cronológicamente en la mismas horas de inicio y fin establecidas en un cronograma programado y previamente establecido con los participantes para que ésta se desarrolle; este aspecto se define a menudo como "asincrónico".

La particularidad <<abierta>> apunta a que la sesión de clase en la que sucede la interacción puede ser accedida por cualquier persona de forma libre sin que medie ningún acuerdo previo determinado a través un procedimiento de inscripción, también quiere decir que el público objetivo a quien apunta una actividad de enseñanza-aprendizaje puede ser diverso, con requisitos muy variados o sin cumplir requisitos establecidos y por último también refiere a que las sesiones de clases pueden ser observadas abiertamente por otros actores distintos de aquellos definidos en el proceso de enseñanza. 
Los expertos consideran que el proceso educativo en la modalidad de teleeducación tiene el mismo grado de validez y alcanza el mismo objetivo de enseñanza, logro de aprendizaje y competencias profesionales que su equivalente tradicional, presencial, sincrónico y cerrado, y que debe ser considerado como la continuación de la educación y no la suplantación del proceso habitual acostumbrado. (Sociedad de la información de Uruguay a través de Educantel, 2020)

Perú fue uno de los primeros países en América Latina en tomar la decisión de establecer un estado de emergencia sanitaria a nivel nacional. El 16 de marzo, a través de un decreto supremo puso a sus ciudadanos en una de las cuarentenas más estrictas del continente para así intentar combatir la propagación del COVID-19. (Decreto Supremo 044-2020, 2020)

Esta actitud fue saludada y en muchos casos aplaudida a nivel internacional. Varios gobiernos latinoamericanos lo tomaron incluso como un punto de referencia regional para el establecimiento de medidas sanitarias en sus respectivos países. (Ipsos. Abril de 2020)

Actuar rápido y declarar un conjunto de restricciones y regulaciones, evitar el contacto físico y el desplazamiento de personas, cerrar las fronteras terrestres, marítimas y aéreas, repatriar a los ciudadanos, reforzar los recursos del sistema de salud a todos los niveles, crear nuevas condiciones hospitalarias, paralizar la actividad productiva prescindible, crear paquetes de ayuda económica a la población más necesitada, entre otras, fueron las medidas tomadas por el Poder Ejecutivo para enfrentar rápidamente la pandemia declarada.

La educación tampoco fue ajena a la afectación por estas restricciones y regulaciones de cuarentena en momentos de emergencia sanitaria. Parte de las acciones tomadas rápidamente en dicha situación fueron: detener de forma temprana la enseñanza en todo el país, suspender las clases a todos los niveles, paralizar el año escolar, cerrar las instalaciones educativas, impedir el desplazamiento de personas a las escuelas, colegios y casas de estudio de cualquier tipo.

Estos esfuerzos del gobierno requirieron sin embargo del apoyo y el compromiso de la sociedad peruana para lograr la efectividad esperada. Perú daba la impresión de estar haciendo las cosas bien, sin embargo, ¿Qué pasó?

La fractura del civismo salió a la luz inmediatamente. En diferentes ciudades y regiones del país se mostraron algunas realidades en el comportamiento de las personas para el cumplimiento de sus deberes como ciudadano, y respetar las leyes y las disposiciones del gobierno e instituciones de la administración y el orden público y contribuir al correcto funcionamiento de la sociedad y al bienestar de los demás miembros de la comunidad.

Se observó una conducta desfavorable en la relación entre los individuos y la convivencia colectiva dentro del marco social, así como la despreocupación y poco cuidado por los intereses de la nación en un sector mayoritario de la ciudadanía. Se sumaron a estas realidades la ignorancia sanitaria de la población, la profunda desigualdad social y los bajos niveles de la economía personal en los sectores socioeconómicos pobres.

La prestación de la educación, como servicio primordial en la sociedad, también se vio impactada. Se volvió un reto para el gobierno defender este derecho humano y mantenerlo disponible durante la cuarentena sin comprometer la salud de los ciudadanos. 
Entonces, ¿por qué este plan macro gubernamental establecido de forma temprana no evitó el avance de los contagiados, hospitalizados y fallecidos por COVID-19 en el país? Muchos analistas encontraron varios factores que ayudaron entender la respuesta a esta pregunta.

\section{- Informalidad.}

En el año 2017 el Informe Anual sobre Producción y Empleo Informal en el Perú reflejó que algo más de dos tercios de la PEA (Población Económicamente Activa), 72,5\%, se dedicaba a la economía informal (Figura 1) (INEI, 2017).

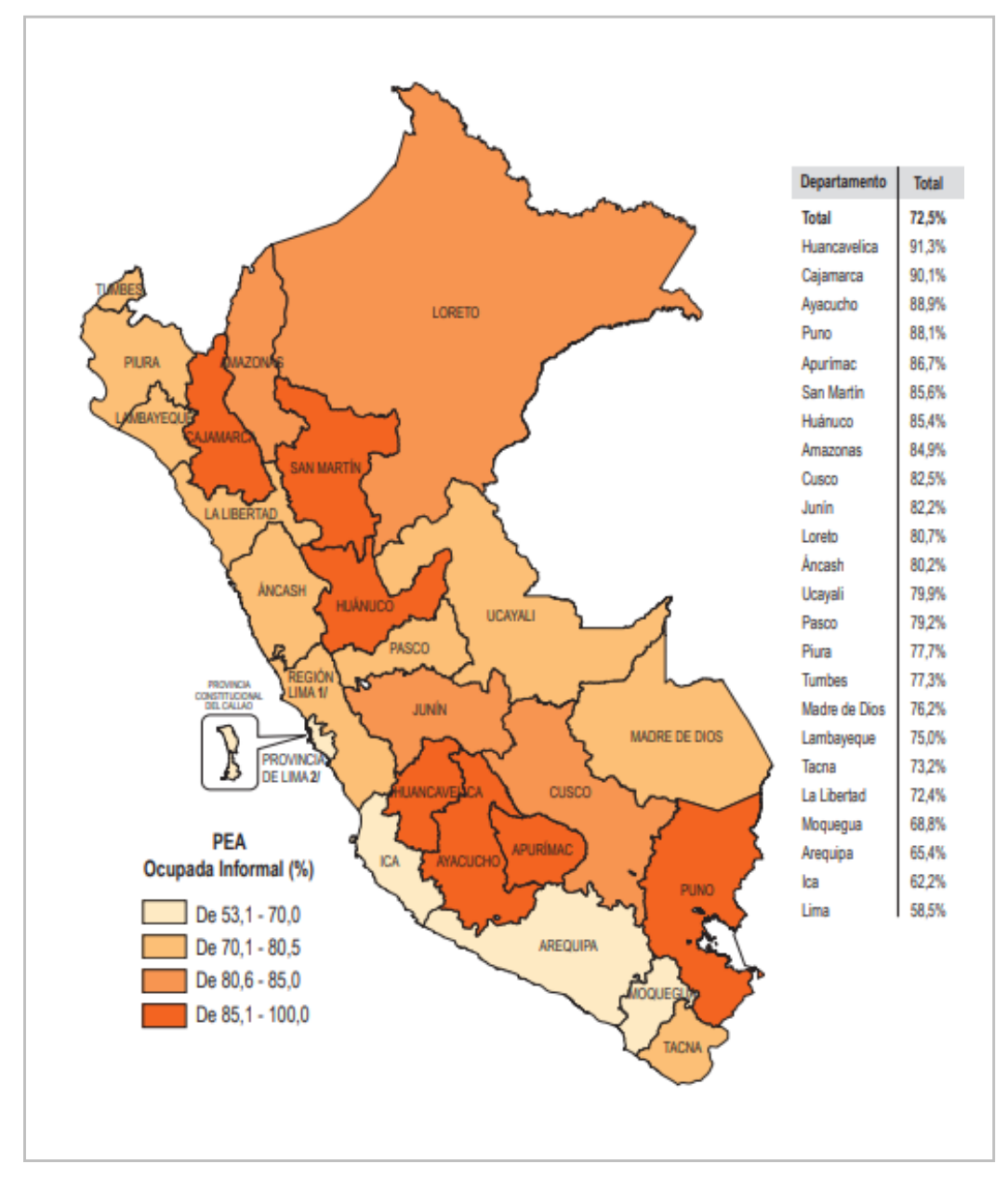

Figura 1. Tasa de informalidad laboral en el Perú. Porcentaje

Fuente: INEI, 2017

Al revisar la Encuesta Nacional de Hogares sobre Condiciones de Vida y Pobreza en el Perú de 2019 se puede apreciar que este indicador mostró una ligera recuperación, 71,1 \% (Figura 2) (PERUCAMARA, 2019). 


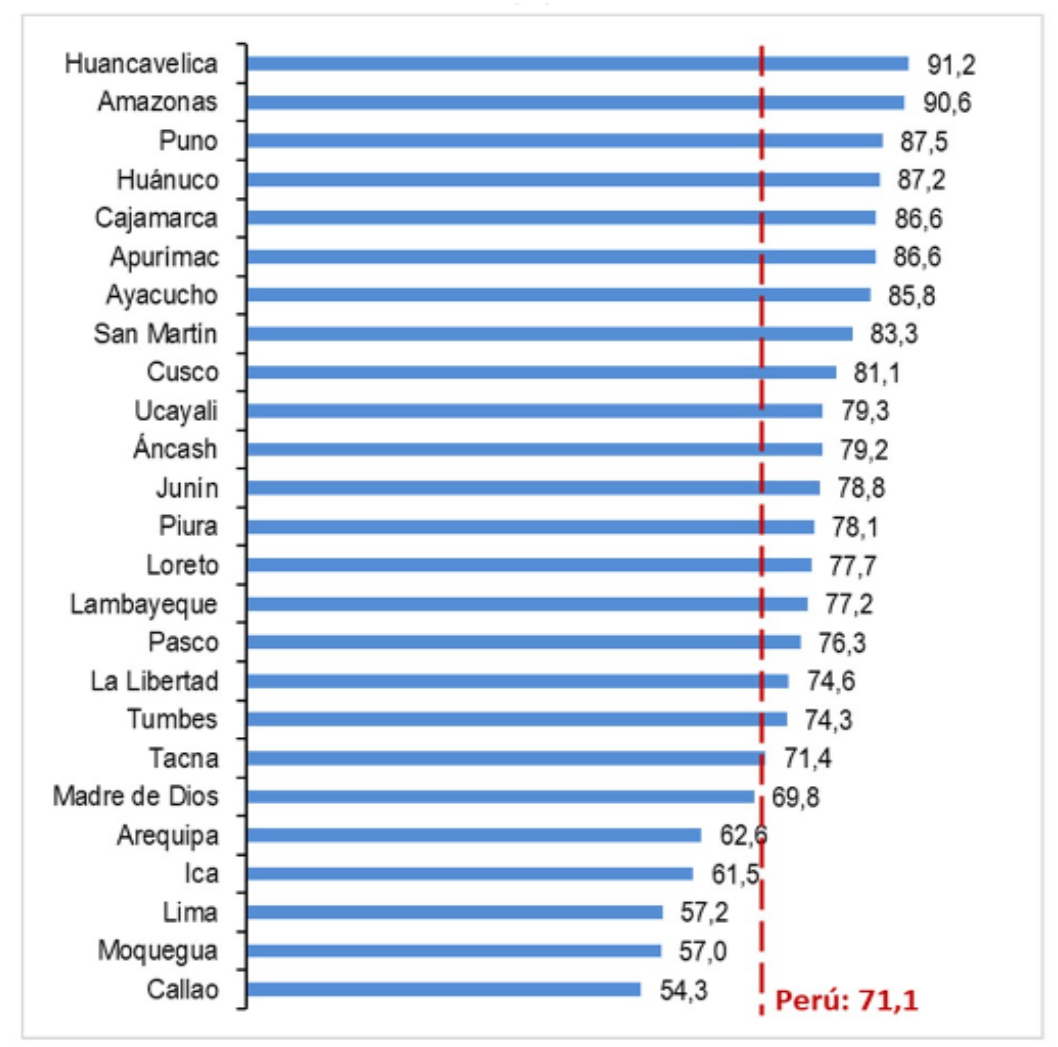

Figura 2. Tasa de informalidad laboral en el Perú

Fuente: PERUCAMARA, 2019

Las personas que trabajan en oficios día por día obtienen sus recursos monetarios y logran sus ingresos un día a la vez, por lo que su pensamiento, modo de vida y gestión en el hogar también tienen el mismo ritmo.

Estos ciudadanos, sustentados en la difícil condición de su económica familiar y la de su hogar, encuentran la necesidad de abandonar sus domicilios pese a las restricciones en las regulaciones establecidas por el gobierno, y salen a trabajar exponiéndose a espacios $y$ ambientes con pocas condiciones sanitarias, para conseguir el dinero necesario con el que luego obtener alimento y recursos de higiene para los miembros de vivienda.

\section{- Poca distancia física en los mercados.}

En el Perú existen más de 2600 mercados de abastecimiento con una expansión de $138 \%$ en los últimos 20 años. El 88,2 \% de ellos son minoristas, lo que implica un comercio hacia el consumidor final y por ende el contacto físico directo con la población (Figuras 3 y 4) (Censo Nacional de Mercados de Abasto, 2016) "...los mercados de abastos continúan siendo la principal forma de suministro de alimentos, bienes y servicios de los consumidores a nivel nacional" (Sánchez, A. 2016). 


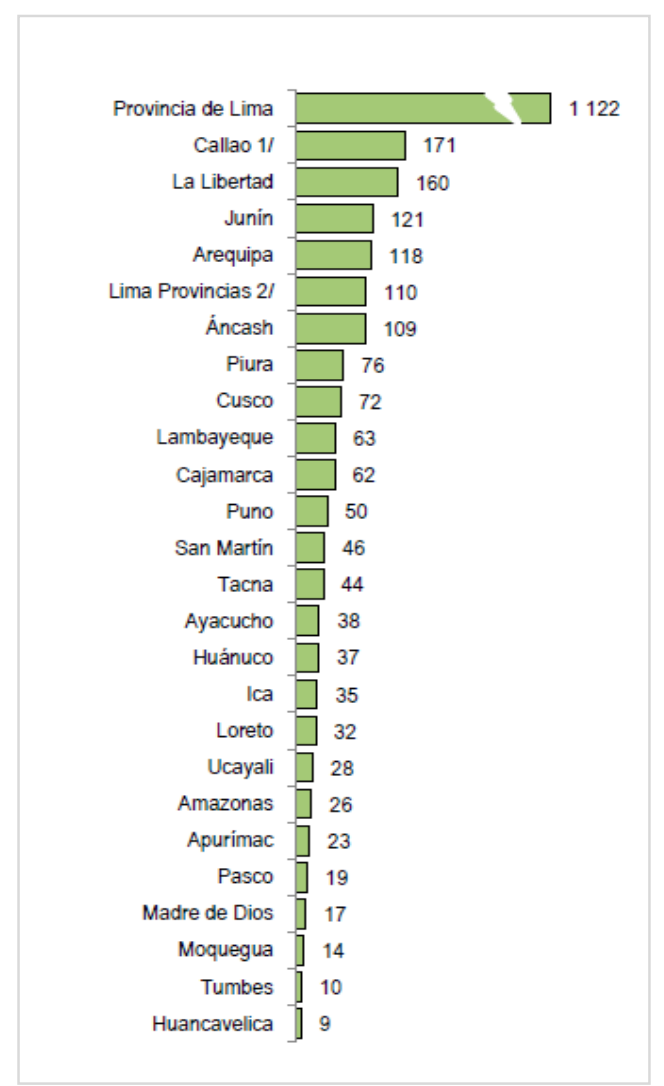

Figura 3. Mercado de Abastos en el Perú. Distribución por Departamento Fuente: CENAMA. 2016

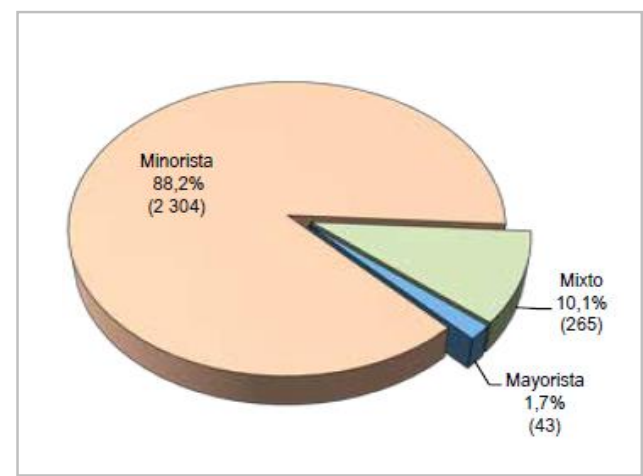

Figura 4. Mercado de Abastos en el Perú. Distribución por Tipo Fuente: CENAMA. 2016 
Los mercados de abastos de alimentos generalmente constituyen sitios de aglomeración de personas. A raíz del establecimiento del estado de emergencia en el país, en el que se definieron horarios reducidos de atención y cierres totales los fines de semana y feriados como parte de las regulaciones indicadas durante la cuarentena, estos mercados se convierten en una fuente de contagio considerable.

El presidente de la república declaró a dichos mercados como principales sitios de contagio para los ciudadanos. "Identificamos que los problemas se estaban dando en las zonas de contagio más concentradas, que eran los mercados. Dijimos: vamos a trabajar directamente con los municipios para revertir la situación", (Presidencia de la República del Perú, 19/05/2020)

En muchos de los mercados se encontró que varios de sus trabajadores resultaron positivo al COVID-19 en pruebas realizadas y estuvieron trabajando días antes en dichas condiciones mientras mantuvieron contacto y poca distancia física con otras personas.
Los ciudadanos al frecuentar estos mercados como compradores se convierten en parte de estas aglomeraciones al desplazarse dentro del amontonamiento caótico y desordenado y se exponen al contagio.

\section{- Bancos.}

Como parte del grupo de medidas tomadas para ayudar a la población que hubiese perdido su empleo a causa de las restricciones establecidas durante el estado de emergencia, el gobierno creó un paquete de ayuda económica dirigido a 6,8 millones de hogares vulnerables. El paquete incluye el pago de bonos monetarios a un miembro de cada uno de estos hogares. (Decreto de Urgencia 053-2020, 2020)

Aunque el ejecutivo implementó formas virtuales para el cobro de dichos bonos, solo un poco más de un tercio aproximadamente de la PEA (Población Económicamente Activa) en el Perú, 42 \%, cuenta con acceso a servicios financieros (Tabla 1) (Encuesta Nacional de Hogares, 2017).

Tabla 1. Inclusión financiera de la PEA en el Perú. Porcentaje

\begin{tabular}{|c|c|c|c|c|c|}
\hline \multirow{2}{*}{$\begin{array}{l}\text { Caracteristicas } \\
\text { demográficas }\end{array}$} & \multicolumn{5}{|c|}{ Acceso a algún servicio financiero $1 /$} \\
\hline & 2015 & 2017 & $\begin{array}{r}\text { Caracteristicas y ámbito } \\
\text { geográfico }\end{array}$ & 2015 & 2017 \\
\hline Total & 38,7 & 42,0 & Condición de pobreza & & \\
\hline Sexo & & & Pobre & 20,9 & 22,7 \\
\hline Hombre & 37,9 & 41,7 & No pobre & 42,6 & 46,1 \\
\hline \multirow[t]{2}{*}{ Mujer } & 39,6 & 42,3 & & & \\
\hline & & & Area de residenica & & \\
\hline \multicolumn{6}{|l|}{ Grupos de edad } \\
\hline 14 a 24 años & 30,7 & 35,2 & Urbana & 43,5 & 46,8 \\
\hline 25 a 44 años & 42,3 & 46,1 & Rural & 22,6 & 24,4 \\
\hline \multirow[t]{2}{*}{45 y más años } & 36,7 & 38,8 & & & \\
\hline & & & Región natural & & \\
\hline \multicolumn{6}{|l|}{ Nivel educativo } \\
\hline Primaria $2 /$ & 20,0 & 22,5 & Costa & 44,0 & 48,8 \\
\hline Secundaria & 30,5 & 33,4 & Sierra & 32,1 & 33,3 \\
\hline Superior no Univ. & 57,4 & 59,2 & Selva & 31,8 & 33,7 \\
\hline Superior Univ. & 73,0 & 76,3 & & & \\
\hline
\end{tabular}

Fuente: Encuesta Nacional de Hogares, 2017 
Muchos de los beneficiarios de estos bonos tienen entonces que dirigirse presencialmente a alguna oficina bancaria a cobrarlos y someterse también a los horarios reducidos de atención y los cierres totales los fines de semana y feriado, hacer largas colas y formar parte de las aglomeraciones que, una vez más, constituyen fuente de contagio.

- Aglomeraciones en el trasporte público. Los ciudadanos que tuvieron que salir a trabajar, comprar en los mercados o realizar trámites en las oficinas bancarias necesitaron movilizarse en transporte público, buses, metro, taxis y otros.

En las estaciones se aglomeran gran cantidad de ciudadanos y hacen largas colas. Igual sucede dentro de los vagones y buses durante el desplazamiento a sus destinos. Algunas de estas personas fueron identificadas positivos al COVID-19 quienes al no mantener la distancia física mínima establecida se convirtieron en fuente de contagio.

- Viviendas con familias numerosas.

Un número considerable de hogares en zonas socioeconómicas pobres en el Perú residen en viviendas hacinadas. En ellas viven 5 personas o más sin habitaciones individuales para dormir, en algunos casos duermen 3 o 4 en un mismo espacio cerrado y donde no existe una higiene y seguridad satisfactorias. Algunos de estos miembros de la familia salen diariamente a trabajar en oficios informales, o asisten a los bancos a cobrar los bonos de ayuda económica o se desplazan en transporte público para tales fines, y regresan luego a sus hogares hacinados aumentando la posibilidad de contagiar a sus familiares si fueron infectados antes.

\section{- Costumbres.}

Perú es un país con una gastronomía tan rica como variada, siendo uno de los sitios más biodiversos del planeta y con una agricultura basta y grande. El ciudadano peruano está acostumbrado a ingerir productos alimentarios frescos, del día si es posible, sobre todo los alimentos que provienen de la tierra, como frutas y verduras, por lo que muchas personas acostumbran a salir con frecuencia a los mercados locales que a veces también son informales, para comprar alimentos de calidad y bajo precio, exponiéndose a aglomeraciones y contagio. Muchos ciudadanos, demuestran civismo y responsabilidad ciudadana y cumplen las regulaciones establecidas por el gobierno peruano para el estado de emergencia, algunos reducen las salidas y la exposición al contagio al mínimo imprescindible, sin embargo, otros no. El individualismo, egoísmo y la ignorancia selectiva sobre lo que sucede alrededor provoca mucho daño. Esto no solo afecta al Perú sino a todo el mundo. Los problemas de fondo que ha descubierto la pandemia no son nuevos y han quedado expuestos de forma brutal y dolorosa.

En la mayoría de los casos estos ciudadanos forman parte de la cadena de personas en las que se puede encontrar un miembro que participa de forma activa o pasiva de la educación en algún nivel. Haberse expuesto al contagio y regresar a las aulas para continuar con el proceso educativo tradicional, presencial, sincrónico y cerrado se vuelve una fuente de contagio muy grande.

Mirando al futuro de corto y mediano plazo es entendible entonces que las personas deben cambiar ciertos comportamientos en la sociedad, y en ellos está la actitud hacia la educación. En este aspecto urge por lo tanto buscar y defender el uso de formas de enseñanza diferente a las tradicionales para mantener a la ciudadanía educada y protegida sanitariamente. La teleeducación se muestra entonces como una alternativa eficaz y de calidad para llenar este vacío. 
Sin embargo, la teleeducación no es ajena a dificultades y barreras. Es justo también indicar que los esfuerzos para lograr eficiencia y eficacia en su ejecución implican que los actores del proceso educativo cumplan ciertos requisitos. Entre ellos se pueden mencionar los más comunes a los que debe prestarse atención primaria:

- Compromiso de los alumnos y profesores para realizar las actividades de enseñanza de acuerdo al diseño pedagógico de forma que se pueda alcanzar el logro de aprendizaje.

- Ambiente contextual, tiempo y espacio para realizar las actividades apropiadamente y con calidad.

- Contar con ciertas tecnologías de la información y las comunicaciones requeridas para ejecutar las actividades con calidad: computadora, laptop, tableta, teléfono inteligente, conexión a internet, recursos multimedia, aplicaciones de uso específico, navegador, entre otros.

- Acceso a medios de comunicación interactivos como radio y televisión de acuerdo al diseño de enseñanza definido.

- Capacidades, conocimiento y habilidades para analizar recursos, descargar información rápidamente e interactuar basándose en textos, imágenes, audios y videos.

- Competencias informáticas para manejar apropiadamente las aplicaciones, software y sistemas de la plataforma tecnológica educativa.

- Soporte tecnológico disponible por parte de la institución educativa para brindar asistencia frente a dificultades durante la realización de las actividades educativas.

Aun así, la teleeducación constituye un reto para muchos profesores, alumnos y personal de soporte, en una sociedad no acostumbrada. Desarrollar habilidades que no habían experimentado anteriormente, autodisciplina, autonomía, confianza, así como adquirir nuevas competencias emocionales se vuelve necesario.

El impacto del COVID-19 en la educación en Perú es significativo y urge enfrentar la situación de forma objetiva y rápida. Se vuelve imprescindible interpretar la oportunidad para dar otra mirada y redefinir los factores de éxito para la supervivencia.

¿Cuáles son los retos que se avecinan para continuar con el servicio educativo en modalidad de teleeducación a nivel nacional?

- Cambiar paradigmas y dar otra mirada a las estrategias de aprendizaje, herramientas e instrumentos para la enseñanza.

- Implementar tele plataformas y brindar recursos eficaces a los profesores, alumnos y personal de apoyo.

- Incrementar y mejorar la calidad del acompañamiento de los padres, tutores y miembros de la familia.

- Incluir el soporte emocional a los alumnos y profesores como parte de la fórmula de la enseñanza.

- Aumentar la intervención del sector a través de las instituciones de la administración y gobierno para renovar los dispositivos legales y aumentar las capacidades de las instalaciones.

La masificación de la teleeducación implica que la inversión en la infraestructura y en los actores del proceso educativos crezca sustancialmente. La Encuesta Nacional a Instituciones Educativas realizado por el INEI en 2018 refleja la realidad del país en este sentido (Tabla 2). 
Tabla 2. Espacios Educativos con Tecnologías Digitales. Porcentaje

\begin{tabular}{l|rr}
\hline \multirow{2}{*}{ Area } & \multicolumn{2}{c}{ Instituciones educativas } \\
\cline { 2 - 3 } Total & Primaria & Secundaria \\
\cline { 2 - 3 } Urbana & $\mathbf{3 3 , 0}$ & $\mathbf{7 2 , 6}$ \\
Rural & 74,4 & 84,4 \\
\hline
\end{tabular}

Fuente: Encuesta Nacional a Instituciones Educativas, 2018

- Solo el 33 \% de las instituciones educativas de nivel primaria y el $72,6 \%$ del nivel secundaria cuentan con espacios físicos con recursos de Tecnología de la Información y las Comunicaciones a ser incorporados en el proceso de enseñanza- aprendizaje.

- Menos de la mitad de los docentes a los niveles inicial, primaria y secundaria ha participado en algún programa de formación en tecnologías digitales (Figura 5).
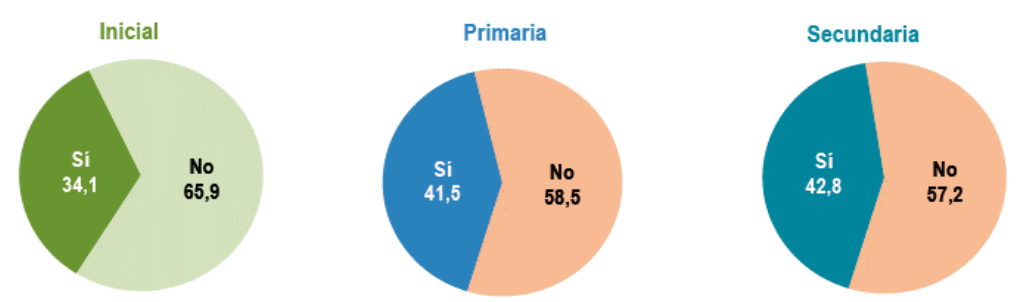

Figura 5. Formación de docentes en tecnologías digitales. Porcentaje

Fuente: Encuesta Nacional a Instituciones Educativas, 2018

Tabla 3. Acceso a internet en Instituciones Educativas. Porcentaje

\begin{tabular}{l|rrr}
\hline \multirow{2}{*}{ Área } & \multicolumn{3}{c}{} \\
\cline { 2 - 4 } & Inicial & Instituciones educativas \\
\cline { 2 - 4 } Total & 12,0 & 24,5 & Secundaria \\
\cline { 2 - 4 } Urbana & 26,2 & 70,5 & 59,2 \\
Rural & 2,7 & 8,9 & 79,5 \\
\hline
\end{tabular}

Fuente: Encuesta Nacional a Instituciones Educativas, 2018

- El acceso a internet en las instituciones educativas es extremadamente bajo en el país. El nivel inicial apenas llega al $12 \%$, el nivel primaria $24,5 \%$ y el nivel secundaria el $59,2 \%$. En los tres niveles el porcentaje es mayor en el área urbana que en la rural (Tabla 3). 
Después de viajar a través de este análisis se puede llegar a la conclusión de que recurrir a modalidades de educación que proporcionen flexibilidad con la ubicación geográfica del alumno y el profesor bajo la que pueden estar en espacios diferentes y distantes entre sí y de la casa de estudios a que pertenecen, así como facilitar a los demás actores del proceso educativo que no compartan el mismo ambiente físico se vuelve prioridad y estratégicamente imprescindible.

De igual forma, aportar conveniencia con la disponibilidad horaria para la ejecución de las actividades de enseñanza-aprendizaje facilita la autonomía de agenda del alumno y el profesor en la gestión de su proceso educativo.

Mejorar la infraestructura de las instalaciones y capacitación de los docentes en Tecnologías de la Información y las Comunicaciones para los recursos digitales se vuelve imprescindible. En momentos de pandemia y crisis sanitaria mundial COVID-19, la teleeducación constituye un medio válido y confiable para la educación como alternativa para generar oportunidades y resultados de igual calidad, extender y mantener los servicios educativos, garantizar la calidad del proceso de enseñanza-aprendizaje y continuar con la formación y el desarrollo de individuos que aporten al bienestar y la prosperidad, en la vida cotidiana de los ciudadanos y así continuar con el objetivo fundamental: el desarrollo integral de la persona en la sociedad.

\section{REFERENCIAS BIBLIOGRÁFICAS}

BBC News Mundo. Edición 22 de mayo de 2020.

Diario oficial El Peruano. Decreto de Urgencia Nro 053-2020 PCM. https:// busquedas.elperuano.pe/normaslegales/ decreto-de-urgencia-que-otorga-un- bono-extraordinario-al-per-decreto-deurgencia-n-053-2020-1866068-1/

Diario oficial El Peruano. Decreto Supremo Nro 044-2020 PCM. https://busquedas. elperuano.pe/normaslegales/decretosupremo-que-declara-estado-deemergencia-nacional-po-decreto-supremon-044-2020-pcm-1864948-2/

INEI, Instituto Nacional de Estadística e Informática de Perú. Censo Nacional de Mercado de Abastos, 2016. https:// www.inei.gob.pe/media/MenuRecursivo/ publicaciones_digitales/Est/Lib1448/libro.pdf

INEI, Instituto Nacional de Estadística e Informática de Perú. Encuesta Nacional de Hogares, Cp 2, Características de la Población Económicamente Activa, 2017. https:// www.inei.gob.pe/media/MenuRecursivo/ publicaciones_digitales/Est/Lib1537/cap02. pdf

INEI, Instituto Nacional de Estadística e Informática de Perú. Encuesta Nacional a Instituciones Educativas, 2018. https:// www.inei.gob.pe/media/MenuRecursivo/ publicaciones_digitales/Est/Lib1684/libro. pdf

INEl, Instituto Nacional de Estadística e Informática de Perú. Informe anual sobre producción y empleo informal en el Perú. 2017. https://www.inei.gob.pe/media/ MenuRecursivo/publicaciones_digitales/Est/ Lib1589/libro.pdf

Ipsos. La Crisis del Coronavirus. Encuesta a líderes de opinión en América Latina. Informe general. Abril, 2020.

Ministerio de Educación del Perú. www.gob. pe/minedu 
PERUCAMARA, Cámara Nacional de Comercio, Producción, Turismo y Servicios Perú, 2019. https://www.perucamaras.org. pe/nt380.html

Presidencia de la República del Perú. Mensaje a la nación del Presidente de la Republica, 19 de mayo de 2020. https://www.youtube.com/ watch?v=JcwNAmGhbj4
Sanchez Aguilar, Anibal, Dr., Jefe del INEI. "Presentación del resultado del Censo Nacional de Mercados de Abastos (CENAMA)", 2016. https://www.youtube. $\mathrm{com} /$ watch?v=Vm-RECETZko

www.educanet.antel.com.uy 